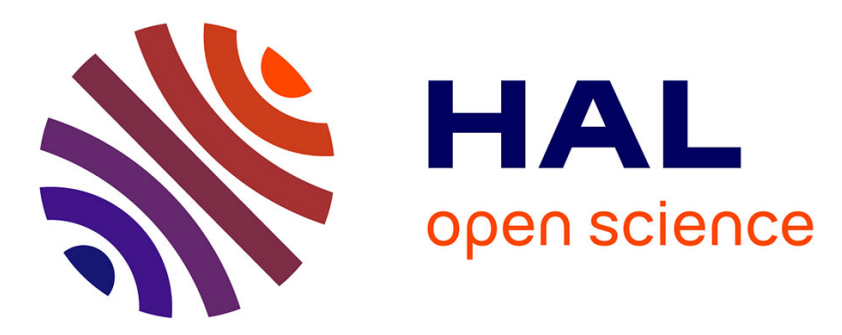

\title{
Les juridictions pénales britanniques et françaises face aux Anti-OGM : au-delà des différences, une communauté d'esprit
}

\author{
Luc Bodiguel, Michael Cardwell
}

\section{- To cite this version:}

Luc Bodiguel, Michael Cardwell. Les juridictions pénales britanniques et françaises face aux AntiOGM : au-delà des différences, une communauté d'esprit. Revue Juridique de l'Environnement, 2011, 36, pp.267-279. hal-01688511

\section{HAL Id: hal-01688511 https://hal.science/hal-01688511}

Submitted on 7 Jan 2022

HAL is a multi-disciplinary open access archive for the deposit and dissemination of scientific research documents, whether they are published or not. The documents may come from teaching and research institutions in France or abroad, or from public or private research centers.
L'archive ouverte pluridisciplinaire HAL, est destinée au dépôt et à la diffusion de documents scientifiques de niveau recherche, publiés ou non, émanant des établissements d'enseignement et de recherche français ou étrangers, des laboratoires publics ou privés. 


\title{
Les juridictions pénales britanniques et françaises face aux Anti-OGM : au-delà des différences, une communauté d'esprit
}

\section{LUC BODIGUEL}

Chargé de Recherche,

\section{Michael CARDWELL}

Professeur à l'Université de Leeds

\author{
Laboratoire droit et changement social, UMR \\ 3128 CNRS/Université de Nantes
}

PRE PRINT : article publié sur Revue Juridique de l'Environnement, 2011/2 Volume 36, 267-279.

\section{Introduction $^{1}$}

Depuis plus de dix ans, le développement des organismes génétiquement modifiés (OGM) développés à des fins alimentaires a été accompagné de fortes protestations ou contestations qui ont pu prendre la forme de destructions matérielles: de l'arrachage symbolique de quelques plants génétiquement modifiés (GM) à la destruction partielle ou totale des cultures prévues à des fins de recherche ou de commercialisation. Au RoyaumeUni, depuis l'an 2000, plus de 54 champs GM ont été l'objet de destruction ${ }^{2}$, ce qui a conduit certains scientifiques à demander une protection des laboratoires et centres de recherche. ${ }^{3} \mathrm{En}$ France, la totalité des champs OGM connus semblent avoir été fauchés ${ }^{4}$ et les actions continuent contre les laboratoires qui poursuivent des essais. ${ }^{5}$ Ces actions risquent de se poursuivre et de se diversifier vu la relance des autorisations d'OGM par la Commission européenne et l'inscription de nouveaux OGM au registre national des semences. ${ }^{6}$

Ces mouvements anti-OGM ont puisé une force nouvelle dans la position de la Cour de justice de l'Union européenne relative à la publicité des lieux de culture. Cette décision était d'autant plus importante que nombre de gouvernements étaient favorables à une

\footnotetext{
${ }^{1}$ Ce travail fait suite à la publication de l'ouvrage collectif suivant: L. Bodiguel and M. Cardwell (eds), The Regulation of Genetically Modified Organisms: Comparative Approache, Oxford University Press, Oxford, 2010, ISBN: 9780199542482 dans lequel le chapitre 1 porte sur « la participation, les préférences et les protestations citoyennes » et la conclusion sur « la désobéissance civile ». Il a fait l'objet d'une présentation lors du colloque de l'Union mondiale des agraristes universitaires (UMAU) à l'Université de Tolède le 8 juin 2010.

${ }^{2}$ V. GM Researcher Despairs as Three Years' Work Lost, Times Higher Education, 7 août 2008.

${ }^{3}$ HJ Atkinson and PE Urwin, Europe needs to protect its transgenic crop research, 453 Nature 979, 19 juin 2008 (453 est le numéro de volume et 976 est le numéro de page). Pour en faciliter les recherches, les auteurs ont choisi de maintenir le format officiel britannique des références anglo-saxonnes.

${ }^{4} \mathrm{G}$ Hayes, Collective action and civil disobedience: the anti-GMO campaign of the Faucheurs Volontaires, (2007) 5 French Politics 293, (v. plus spécifiquement p.296).

5 Arrachage de pieds de vigne OGM le 15 août 2010 sur une parcelle expérimentale de l'INRA : http://Actuenvironnement.com (consulté le 13 septembre 2010).

${ }^{6}$ Autorisation de commercialisation de la Commission concernant la pomme de terre Amflora (BASF) et de plusieurs variétés du maïs MON 863: http://www.lemonde.fr/europe/article/2010/03/02/la-commissioneuropeenne-s-apprete-a-autoriser-la-commercialisation-d-ogm_1313163_3214.html (consulté le 20 septembre 2010) ; 38 nouvelles variétés de maïs OGM inscrites en France : http://Actu-environnement.com (consulté le 13 septembre 2010).
} 
planification secrète de cultures GM. En Grande-Bretagne, le débat a été vif ${ }^{7}$ mais c'est à partir d'une question préjudicielle du Conseil d'Etat français que la Cour européenne a mis fin à toute équivoque : la localisation des lieux de dissémination volontaire d'OGM dans l'environnement ne peut plus être tenue confidentielle pour des raisons d'ordre public. ${ }^{8}$

En France, sur la base des articles 25 et 31 de la directive européenne 20001/18 ${ }^{9}$, le législateur a fini par prendre explicitement acte de cette interprétation ${ }^{10}$ puisque le détenteur d'une autorisation concernant un OGM « doit déclarer auprès de l'autorité administrative les lieux où sont pratiquées ces cultures », « doit également informer, préalablement aux semis, les exploitants des parcelles entourant les cultures d'OGM » et que le ministère «établit un registre national indiquant la nature et la localisation des parcelles culturales d'OGM », à charge pour les préfectures d'en assurer la publicité «par tous moyens appropriés, notamment sa mise en ligne sur l'internet. ${ }^{11}$

De même, au Royaume-Uni, les demandes d'autorisations de dissémination en plein champ $^{12}$ doivent préciser les lieux de culture pressentis et les registres anglais, écossais, gallois et irlandais du nord doivent inclure, inter alia, la localisation de tout champ OGM légalement autorisé. ${ }^{13}$

Inévitablement, ces destructions systématiques ont conduit à la saisine des juridictions pénales. Lors des procès, les juges ont alors dû se prononcer sur la nature des infractions commises et sur le niveau des sanctions. Dans l'ensemble, la nature et la constitution de l'infraction ne fait pas de doute (destruction volontaire de bien d'autrui selon la formulation française) ; la question de la sanction est plus difficile à traiter vu les incertitudes tenant aux dangers éventuels des OGM. Les juges auraient pu «excuser» les auteurs d'infraction ou se borner à des condamnations symboliques dans l'attente de connaissances scientifiques plus approfondies et vu le contexte politique et philosophique des actions anti-OGM (actions revendiquées comme des actes de désobéissance civile). L'analyse de ces décisions fait plutôt apparaitre les juges comme les tenants d'une vision légaliste; cette constatation vaut principalement pour la France où on a pu assister à un durcissement progressif des condamnations pénales (2) alors que les juridictions britanniques ont pris des positions plus contrastées (1).

${ }^{7}$ v., A Grice, Government to Defy Critics with Secret GM Crop Trials, Guardian, 17 novembre 2008 ; v. aussi la réponse equivoque du gouvernement britannique sur l'existence de ces plans: J Riley, DEFRA Responds to Secret GM Crop Trial Claim, Farmers Weekly Interactive, 17 novembre 2008.

${ }^{8}$ Affaire Commune de Sausheim v Azelvandre C-552/07, 17 février 2009.

${ }^{9}$ Directive 2001/18/CE du Parlement européen et du Conseil du 12 mars 2001 relative à la dissémination volontaire d'OGM dans l'environnement et abrogeant la directive 90/220/CEE du Conseil, JO n ${ }^{\circ} \mathrm{L}_{106} \mathrm{du}$ 17/04/2001, 1-39.

${ }^{10}$ Le Conseil d'Etat avait déjà suivi l'interprétation de la Cour: CE, 9 déc. 2009, n²80969, Commune de Sausheim.

${ }^{11}$ Article L. 663-1 c. rur.

${ }^{12}$ Il existe différentes législations pour l'Angleterre, l'Irlande du nord, l'Ecosse et le Pays de Galles. En Angleterre, le dispositif principal relève du Genetically Modified Organisms (Deliberate Release) Regulations 2002, S.I. 2002 No. 2443 (Schedule 1, Part V and Schedule 2, Part III). La législation officielle du Royaume-Uni peut être trouvé sur le site suivant: http://www.opsi.gov.uk/legislation/about legislation.

${ }_{13}$ Pour l'Angleterre, v. Article 34(8) du Genetically Modified Organisms (Deliberate Release) Regulations 2002, S.I. 2002 No. 2443 : l'information doit être donnée au Secretary of State for Environment, Food and Rural Affairs. 


\section{Royaume-Uni : des condamnations contrastées}

A titre préliminaire, il faut souligner une différence fondamentale entre la France et le Royaume-Uni : alors qu'en France, la destruction des champs OGM relève généralement des délits et par conséquent des tribunaux correctionnels composés uniquement de magistrats professionnels, le Royaume-Uni permet dans certains cas de saisir la Crown Court compétente pour les infractions pénales importantes. A l'image de la Cour d'assise, la Crown Court est présidée par un juge professionnel, mais la décision est laissée à l'appréciation d'un jury de douze citoyens. Or, les jurys sont dans l'ensemble plus accessibles aux arguments populaires relatifs notamment aux préoccupations environnementales et sanitaires. ${ }^{14}$

\section{1 - Des jurys favorables aux argumentaires anti-OGM}

Dans le domaine des OGM, la présence d'un jury est recherchée afin de bénéficier d'une justice plus proche des revendications anti-OGM.

Trois affaires sont représentatives à ce titre. Deux concernent directement les OGM, la dernière le changement climatique.

A la suite de la «décontamination » d'un champ GM ${ }^{15}$, Lord Peter Melchett et vingtsept autres volontaires de Grenpeace ont été inculpés de vol et de destruction de biens (criminal damage) devant la Crown Court de Norwich. Acquitté pour le vol le 19 avril $2000^{16}$, le jury les a acquittés de la seconde charge le 20 septembre 2000 considérant que leurs actions étaient justifiées par le besoin de protéger la propriété, en l'espèce les cultures nonOGM avoisinantes. ${ }^{17}$

Une seconde illustration de l'influence potentielle d'un jury peut être apportée avec l'affaire $R v$ Colchester Justices, ex parte Abbott. ${ }^{18}$ En l'espèce, afin d'ouvrir la possibilité d'un procès devant un jury et, selon toute attente, de bénéficier d'une audience plus favorable, les militants anti-OGM ont motivé leur recours par l'existence de dommages causés aux champs OGM supérieurs à ceux qu'invoquaient l'accusation (£13.900 contre £3250). ${ }^{19}$ Leur argumentaire financier n'a pas porté ses fruits ; les Magistrates, puis en appel, la Divisional Court l'ont réfuté. Cependant, cet échec ne doit pas faire oublier le fait que les anti-OGM ont pris le risque de sanctions plus importantes en saisissant la Crown Court pour avoir l'opportunité de présenter leur affaire devant douze représentants de la société civile. Certains commentaires relèvent d'ailleurs le caractère paradoxal de cette logique processuelle : un procès devant la Crown Court devrait normalement profiter à l'accusation puisque la cour peut décider de peines plus sévères et que des peines plus sévères pourraient être appropriées

\footnotetext{
${ }^{14}$ Les verdicts des jurys ne sont pas publiés dans des recueils officiels. On en trouve trace dans les journaux essentiellement.

${ }^{15}$ Terme utilisé par les militants anti-OGM pour parler d'une destruction de cultures GM, celles-ci pouvant « contaminer » les cultures non GM.

${ }^{16}$ Greenpeace Press Release, Jury Fails to Convict Greenpeace Volunteers, 19 avril 2000 (disponible sur http://www.greenpeace.org.uk/media/press-releases/greenpeace-volunteers-cleared-of-theft-in-lyng-gm-trial, consulté le 3 octobre 2010). V., plus généralement, M Stallworthy 'Damage to crops - Part 1' (2000) 150 New Law Journal 728 et 'Damage to crops - Part 2' (2000) 150 New Law Journal 801; et S Tromans and C Thomann, 'Environmental protest and the law' [2003] Journal of Planning and Environmental Law 1367.

${ }^{17}$ Greenpeace Press Release, 28 Greenpeace Volunteers Acquitted in GM Trial, 20 septembre 2000 (disponible sur http://www.greenpeace.org.uk/media/press-releases/28-greenpeace-volunteers-acquitted-in-gm-trial (consulté le 3 octobre 2010).

${ }^{18}$ [2001] EWHC Admin 136; Times, 13 mai 2001; v. aussi [2001] Criminal Law Review 564.

${ }^{19}$ Le seuil financier pour bénéficier d'un procès devant jury s'élève en principe à $£ 5000$. Pour de plus amples détails sur l'affaire, v. Magistrates' Court Act 1980, s 22 (v. supra n. 12).
} 
pour des faits qui ne se limitent pas à la destruction de cultures mais constituent surtout des atteintes à la recherche en matière d'agriculture biotechnologique. ${ }^{20}$

L'ouverture « citoyenne » aux préoccupations environnementales révélée par ces deux affaires pourraient être encore plus large si l'on en croit une décision récente (2008) sur le changement climatique : six militants de Greenpeace, qui avaient escaladé la cheminée de la Centrale électrique de Kingsnorth (charbon) et l'avaient barbouillée de peinture, ont été inculpés pour avoir causé un dommage évalué à 30.000 livres. Devant la Crown Court, ils ont invoqué la nécessité immédiate de protéger la propriété dans le monde du fait de l'impact des activités concernées sur le changement climatique ${ }^{21}$, argument que le jury accepté. D'un point de vue strictement juridique et politique, certains pourraient s'inquiéter de la portée de cette décision. Il est vrai qu'elle peut être analysée comme une tentative d'usurpation du pouvoir législatif dans le domaine de la politique énergétique. Cependant, elle peut aussi être perçue comme un instrument d'équilibrage et de contrôle démocratique : même si les arguments sont difficiles à soutenir légalement, ils correspondent à l'opinion de la population au RoyaumeUni. En outre et surtout, il ne faut pas en exagérer la portée et en avoir peur car, constitutionnellement, à la différence de la décision des juges, elle n'a pas l'autorité d'un precedent, ce qui permet à toute autre juridiction saisie des mêmes faits de trancher indépendamment de la présente décision. ${ }^{22}$

Il n'est pas dit que cette décision ait un impact dans le domaine des OGM, mais elle vient confirmer la sensibilité des jurys et l'intérêt, lorsque cela est possible, d'engager des actions devant la Crown Court.

La sensibilité des juges professionnels est, quant à elle, moins développée...

\section{2 - Les juges professionnels : une position plus sévère}

Si les juges professionnels britanniques rejettent généralement les arguments des faucheurs, ils ont pu leur être favorables en raison de l'absence partielle d'éléments constitutifs des infractions invoquées. Ainsi, dans l'affaire Tilly $v$ Director of Public Prosecutions tranchée par la High Court, le prévenu étaient clairement accusé d'une violation de propriété aggravée (aggravated trespass). ${ }^{23}$ Cette infraction est constituée lorsqu'une personne pénètre illégalement sur des terres en plein jour (in the open air) sur lesquelles des personnes se livrent ou sont sur le point de se livrer à une activité licite, et fasse quelque chose qui a pour effet : (a) d'intimider les personnes ou l'une d'elles afin de la ou de les dissuader de se livrer à cette activité ; (b) de faire obstacle à l'activité, ou (c) de perturber cette activité. ${ }^{24}$ Sans hésitation, le juge a conclu que l'infraction n'était pas réalisée car elle supposait la

${ }^{20}$ «[i]f an expensive experiment is being lawfully conducted in the public interest, may not its destruction demand something more than a mere summary conviction and a relatively trivial penalty ? »: v. [2001] Criminal Law Review 564, 565.

21 V. Greenpeace UK, Breaking News: Kingsnorth Six Found Not Guilty (http://www.greenpeace.org.uk/blog/climate/kingsnorth-trial-breaking-news-verdict-20080910, consulté le 3 octobre 2010).

${ }^{22}$ V. T Brooks, A defence of jury nullification (2004) 10 Res Publica 401 (v. en particulier p. 418).

${ }^{23}$ [2001] EWHC Admin 821; Times, 27 November 2001; v. aussi [2002] Criminal Law Review 128.

24 «Aggravated trespass » est une infraction spécifique relevant du Criminal Justice and Public Order Act 1994 (Section 68(1) dans sa version alors en vigueur (v. supra site internet législation officielle). «trespasses on land in the open air and, in relation to any lawful activity which persons are engaging in or are about to engage in on that or any adjoining land in the open air, does there anything which is intended by him to have the effect (a) of intimidating those persons or any of them so as to deter them or any of them from engaging in that activity; (b) of obstructing that activity, or (c) of disrupting that activity ». 
présence des personnes «intimidées » ou empêchées de faire quelque chose ${ }^{25}$ et que, par conséquent, la simple destruction de culture n'était pas suffisante pour condamner les faucheurs OGM au titre d'aggravated trespass.

De même, les juges professionnels ont pu relever dans un premier temps des « excuses » au bénéfice des faucheurs. Il s'agissait d'une action pour «aggravated trespass » contre des militants qui s'étaient attachés à des tracteurs dans l'intention de perturber le semi de maïs GM. ${ }^{26}$ Bien que convaincus qu'ils étaient des «aggravated trespassers », le District Judge a énoncé que les prévenus avaient des croyances sincères et véritables sur les dangers des cultures GM, qu'ils avaient de véritables craintes pour les terres environnantes, qu'ils avaient des motifs raisonnables pour fonder leurs croyances et leurs craintes compte tenu de leurs connaissances scientifiques concernant les essais de cultures GM et de leur connaissance des lieux. Le juge a aussi mentionné que les prévenus avaient agi avec «toute leur bonne volonté » et n'étaient pas allés plus loin que ce qui était absolument nécessaire pour essayer d'empêcher les semis des cultures. En conséquence, leurs actions ne pouvaient pas être sanctionnées sur le fondement de «l'aggravated trespass» et les faucheurs pouvaient légitimer leur action par la «défense de la propriété selon la common law » (defence at common law of defence of property). On voit ainsi que l'argument de la protection de la propriété peut parfois être retourné : il protège soit les semeurs d'OGM des violations et destructions, soit les agriculteurs exploitants des parcelles avoisinantes non OGM des risques de « contamination ».

Toutefois, malgré ces quelques ouvertures, les juges professionnels sanctionnent sévèrement les destructions de cultures GM.

En premier lieu, dans les deux affaires évoquées, la réussite des anti-OGM est limitée. Ainsi, dans Tilly v Director of Public Prosecutions, même s'il n'a pas retenu l'infraction, le juge a précisé qu'il existait des possibilités d'engager des poursuites pénales sur d'autres fondements qui ne nécessitaient pas la présence ou la présence imminente de cette destruction. ${ }^{27}$ En outre, dans Director of Public Prosecutions v Bayer, les inculpés n'ont pas pu se réjouir de la décision car, en appel, la Divisional Court $^{28}$ a rappelé qu'il n'est possible d'invoquer ces motifs (bonne foi, crainte véritable...) que contre des actes illégaux ou criminels. Or, en l'espèce, la culture du maïs GM était légale (autorisée) et les défendeurs le savaient ; par conséquent, ils n'ont pas pu bénéficier de la défense de la propriété selon la common law. ${ }^{29}$

En second lieu, d'autres décisions de justice refusent clairement les motifs des faucheurs. Dans l'affaire Monsanto PLC v Tilly traitée par la Court of Appeal ${ }^{30}$, la majorité des personnes mises en cause étaient membres de GenetiX Snowball, une organisation nongouvernementale qui fait activement campagne contre les cultures GM. Leur «charte d'adhésion » (Handbook) prône des protestations non-violentes et le caractère symbolique des

\footnotetext{
25 [2001] EWHC Admin 821 "presence is necessary before an offence under this section can be made out » [25], ce qui correspond à une " situation in which people are meant to be intimidated, or cannot get on with what they are entitled to do » ibid. [26].

${ }^{26}$ Director of Public Prosecutions v Bayer. Sur les débats suite au jugement du District Judge, v. [2003] EWHC 2567 (Admin); [2004] 1 'Weekly Law Reports' (WLR) 2856 per Brooke LJ; et [2004] Criminal Law Review 663.

${ }^{27}$ V, [2002] Criminal Law Review 128 : un commentateur suggère une action pénale sur la base du Criminal Damage Act 1971.

${ }^{28}$ Ibid (n. 27).

${ }^{29}$ Comme le District Judge a omis cet élément, la décision a été infirmée (v. n. 27).

${ }^{30}$ Times, 30 novembre 1999. Ne pas confondre cette affaire avec celle précitée Tilly v Director of Public Prosecutions.
} 
arrachages de plants GM, les adhérents s'engageant notamment à ne pas détruire plus de cent plants par jour et par personne. ${ }^{31}$ Cet engagement (Pledge) renvoie directement aux définitions de la désobéissance civile que l'on peut trouver dans la doctrine : le fait d'agir en plein jour correspond à l'exigence d'un «acte public» suivant la définition de la désobéissance civile de Rawls dans son ouvrage A Theory of Justice. ${ }^{32}$ De même, la nonviolence fait partie des critères posés par Rawls ; or, la Court of Appeal dans Monsanto PLC $v$ Tilly n'a pas été pas convaincue que l'arrachage des plans satisfaisait à cette exigence, même si les actions étaient symboliques. Pour elle, ces actions «militantes »n'ont aucune portée matérielle et ne peuvent justifier la destruction des OGM. S'il y avait eu un danger immédiat pour le voisinage, l'éradication de la totalité de la culture GM aurait été exigée, mais, selon les juges, il s'agissait seulement de médiatiser une « une bonne cause » pour compenser le fait que les défendeurs étaient incapables de modifier la politique gouvernementale par la seule force de leurs arguments. Autrement dit, «briser la loi » est perçue comme le moyen de se présenter en martyr ${ }^{33}$ et de générer une large publicité à la cause anti-OGM, ce qui est particulièrement mal perçu par le juge britannique.

Notons que, à l'inverse, même lorsque le procureur avance le même argumentaire sur la publicité lors du procès Lord Peter Melchett and the other Greenpeace volunteers de 2000, les jurys continuent à ne pas reconnaitre la culpabilité. ${ }^{34}$ Cette solution confirme l'importance de la stratégie procédurale (jury ou magistrats professionnels) et l'avantage que les anti-OGM peuvent tirer d'une action devant la Crown Court. ${ }^{35}$

Les fondements de la décision Monsanto PLC v Tilly méritent d'être précisés. Dans cette affaire, la Court of Appeal se réfère en particulier à deux décisions pour établir les circonstances légitimant des actions de destruction.

D'une part, ils visent le cas Burmah Oil Co Ltd v Lord Advocate de la House of Lords dans lequel les juges devaient décider s'il y avait lieu d'accorder une indemnisation à une compagnie pétrolière suite à la destruction de ses installations en Birmanie par l'armée britannique. Le problème venait $\mathrm{du}$ fait que ces destructions étaient survenues dans «l'urgence de la guerre » afin d'éviter que les installations ne tombent aux mains des forces japonaises. ${ }^{36}$ Finalement, il a été décidé que la compagnie pouvait être indemnisée puisque, même si les faits s'étaient déroulés dans «l'urgence de la guerre », l'armée n'était pas pour autant autorisée à «détruire les biens d'autrui dans l'intérêt public ». De cette décision, il ressort que nul n'a le droit de détruire la propriété d'un autre au nom de l'intérêt public (entendu dans le sens de "protéger les autres d'un danger»), sauf dans des circonstances particulièrement strictes. Dans Monsanto PLC v Tilly, la Court of Appeal semble avoir pensé que les circonstances dans le cas des OGM étaient beaucoup moins extrêmes...

Sur le fondement de la décision Southwark Borough Council v Williams ${ }^{37}$, la Court of Appeal a affiné son raisonnement sur les circonstances légitimant une action de destruction : d'une part, le danger doit être immédiat et évident ; d'autre part, une personne raisonnable

\footnotetext{
${ }^{31}$ Sur le GenetiX Snowball Handbook, v. http://www.gene.ch/pmhp/gs/handbook.htm (consulté le 3 octobre 2010).

32 J Rawls, A Theory of Justice, Oxford University Press (Revised edition), Oxford, 1999, 320.

${ }^{33}$ Le terme de martyr a été largement employé lorsque les anti-OGM ont été condamnés. V. Sheriff Has Made Our Dad a Martyr, Say Children of Jailed GM Protestor, Aberdeen Press and Journal, 20 mars 2000.

${ }^{34}$ V. par ex. M McCarthy, Lord Melchett and Company Back in the Dock to Deny GM Crop Damage, Independent, 5 September 2000.

${ }^{35}$ V. Supra (1.1).

${ }^{36}$ [1965] Appeal Cases (AC) 75 (Rapport officiel de la House of Lords).

${ }^{37}$ [1971] 1 Ch 734 v. supra n. 12).
} 
devrait avoir conclu au vu de la situation que la seule option était la violation de propriété. ${ }^{38}$ Elle insistait aussi - et c'est important - sur le fait qu'une organisation qui fait campagne pour changer une politique gouvernementale doit tendre à le faire par des moyens légaux et non par des procédés illégaux ; or, dans les circonstances de l'affaire Monsanto PLC v Tilly, il existait des possibilités d'actions judiciaires contre les autorisations d'OGM. Il faut cependant souligner que les membres de GenetiX Snowball n'ont pas été encouragés par la Court of Appeal à initier de telles actions, ce qui conforte l'idée selon laquelle des actions en justice contre les autorisations d'OGM risquent d'échouer. ${ }^{39}$

L'analyse de la jurisprudence britannique montre donc que les juges professionnels ont une lecture beaucoup plus légaliste que les jurys. Ce constat est d'autant plus important que les décisions des jurys ont moins de portée juridique. En fait, à l'exception de l'affaire Tilly v Director of Public Prosecutions, et District Judge in Director of Public Prosecutions v Bayer, l'interprétation de la « loi » par les juges conduit à condamner quasi systématiquement les militants anti-OGM, faucheurs ou "empêcheurs de semer » au motif de l'absence de danger ou de circonstance justifiant une action illégale et de l'existence d'autres voies de recours. En ce sens, ils sont proches du raisonnement des juges français.

\section{France}

En France, la distinction entre des affaires soumises à des juges ou présentées devant un jury ne se pose pas. C'est la magistrature professionnelle qui est systématiquement saisie des actions de destructions de plants GM sur des parcelles privées ou publiques, réalisées principalement par les militants - dits «faucheurs volontaires »- anti-OGM. ${ }^{40}$ Comme dans le cas du Royaume-Uni, ce fait est d'importance primordiale : les juges appliquent la loi - en l'espèce la loi pénale - à la lettre et rejettent les arguments des faucheurs. Ce faisant, à la différence des jurys populaires britanniques, ils bannissent du droit les actions qualifiées par leurs auteurs de désobéissance civile.

\subsection{Application littérale du droit pénal.}

Le 26 février 2008, la Cour d'appel d'Orléans a rendu une décision très attendue et désormais bien connue ${ }^{41}$ qui illustre parfaitement la position des juges français. Dans cette affaire, Monsanto, fort de l'autorisation de mise en culture des OGM en cause, réclamait la condamnation des faucheurs volontaires pour destruction de plants génétiquement modifiés et la réparation de son préjudice évalué à 313.108 euros. De leur côté, les «faucheurs » ne remettaient pas en cause la réalisation du délit, au contraire, ils le revendiquaient mais invoquaient à titre principal leur irresponsabilité pénale. Les juges ont fait droit au demandeur : ils ont reconnu le délit de destruction du bien d'autrui commis en réunion visé à l'article L.322-1 du code pénal ${ }^{42}$ et condamné les prévenus à des peines d'emprisonnement

\footnotetext{
${ }^{38}$ Dans Monsanto PLC v Tilly, la Court of Appeal a établi que les actions anti-OGM pouvaient relever à la fois de l'incrimination (Common Law) de «trespass to land» (entrée illégale sur les terres où Monsanto a un intérêt «suffisant» à cultiver) et de celle de «trespass to goods» (destruction des cultures elles-mêmes, une fois détachées de la terre).

${ }^{39}$ Sur une action réussie contre une autorisation OGM, v. $R v$ Secretary of State for the Environment, ex partent Watson [1999] Env LR 310 (Rapport officiel).

${ }^{40}$ Parfois, on a pu voir des opérations plus spectaculaires comme le démontage d'un McDonalds le 11 août 1999.

${ }^{41}$ Les « anti-OGM » espéraient un changement de jurisprudence dans la lignée du moratoire interdisant le MON 810, revirement qui aurait pu influer sur le projet de loi relatif aux OGM alors discuté au Parlement.

${ }^{42}$ Depuis la Loi $n^{\circ} 2008-595$ du 25 juin 2008 relative aux OGM, JORF n0148 du 26 juin 2008 NOR: DEVX0771876L, le nouvel article L.671-15 du code rural rehausse le niveau des sanctions prévues en droit
} 
avec sursis de 2 à 4 mois et à des amendes de 1500 à 3000 euros. En revanche, la peine complémentaire prévue par les premiers juges consistant en une privation des droits civiques n'a pas été confirmée car jugée inadaptée «à une situation où seul le comportement civique des intéressés est en cause ».

\subsection{Rejet de l'irresponsabilité pénale des faucheurs.}

Face à l'action de Monsanto, les faucheurs ont demandé au juge de reconnaitre leur irresponsabilité pénale parce qu'ils auraient agi en raison d'un "état de nécessité », disposition selon laquelle «n'est pas pénalement responsable la personne qui, face à un danger actuel ou imminent qui menace elle-même, autrui ou un bien, accomplit un acte nécessaire à la sauvegarde de la personne ou du bien, sauf s'il y a disproportion entre les moyens employés et la gravité de la menace $»^{43}$

Comme la grande majorité des juges ayant eu à trancher des affaires similaires, la Cour d'Orléans a rejeté ce motif. Se faisant, elle confirme la jurisprudence constante ${ }^{44}$ selon laquelle «le fait justificatif de l'état de nécessité est impuissant à neutraliser l'incrimination de destruction du bien d'autrui ${ }^{45}$ puisqu'il n'existe pas de danger réel et actuel, que l'acte n'est pas nécessaire à la sauvegarde d'une personne ou d'un bien et qu'il n'y a pas de proportionnalité entre les moyens employés et la gravité de la menace.

Le motif central des juges tient à l'absence de danger du fait de l'impossibilité de le caractériser «en l'absence de réponse définitive de la part des scientifiques ». Les juges vont même plus loin en affirmant que le risque de «contamination » lui-même n'est démontré ni en général (les conséquences des OGM sur les autres formes d'agriculture) ni en particulier (des parcelles in concreto contaminée). L'arrêt vient ainsi conforter le refus d'ouvrir l'état de nécessité aux cas où le danger n'est que potentiel ou hypothétique. En d'autres termes, l'arrêt de la Cour d'appel d'Orléans reprend l'idée selon laquelle une «crainte qui (...) n'est fondée

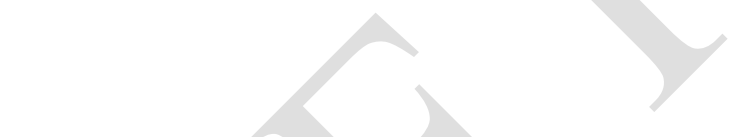

commun; les peine peuvent aller jusqu'à deux ans d'emprisonnement et 75000 euros d'amende ; notons que la peine peut être alourdie si les OGM détruits ont été autorisés pour la recherche.

${ }^{43}$ Etat de nécessité visé à l'article L122-7 du code pénal. Notons que les personnes suspectées de dégradation de champ d'OGM invoquent aussi parfois la légitime défense visées à l'article 122-5 du code pénal ; v. Tribunal de grande instance d'Orléans du 9 décembre 2005, n²345/S3//2005; v. Feldman Jean-Philippe, Les « faucheurs d'OGM » et la Charte de l'environnement (Recueil Dalloz, 2006, 814. Ce motif n'a pas été présenté devant la Cour d'appel Orléans.

${ }^{44}$ Jurisprudence initiée par la Chambre criminelle de la Cour de Cassation du 19 novembre 2002 n $^{\circ} 02-80788$ (note Mayer, Dalloz 2003). Jurisprudence poursuivie notamment par : Cour de Cassation Chambre criminelle, 4 avril 2007, $n^{\circ}$ 06-80.512 ; Cour de Cassation, Chambre criminelle, 7 février $2007 \mathrm{n}{ }^{\circ} 06-80.108$ (v. note Feldman Jean-Philippe, Dalloz 2007, 1310) ; Cour d'appel de Versailles, 22 mars 2007, n06/01902 ; Cour de Cassation, Chambre criminelle, 28 avril 2004, $n^{\circ} 03-83783$, Cour de Cassation, Chambre criminelle, 18 février 2004, $\mathrm{n}^{\circ} 03$ 82951 (v. note Trouilly Pascal, Revue Environnement, juillet 2004, 22). Certaines juridictions de première instance ont tenté de modifier la position de la jurisprudence. Ainsi, le tribunal correctionnel de Versailles (12 janvier 2006) a considéré qu'il existait un état de nécessité vu l'impossibilité de se protéger contre les dangers des mises en culture d'OGM. V. aussi tribunal correctionnel d'Orléans du 9 décembre 2005 (2345/S3//2005, société Monsanto c/ Dufour et a.; RD rur.n³39, 2006, comm. 36.) qui a relaxé 49 faucheurs volontaires sur la base l'état de nécessité. Sur ce dernier jugement, v. Monteillet, op. cit. ; Feldman Jean-Philippe, Les « faucheurs» fauchés par la Cour de cassation, Recueil Dalloz 2007 p. 1310 ; Gossement Arnaud, Le fauchage des OGM est-il nécessaire? Réflexions sur la relaxe des faucheurs volontaires par le tribunal d'Orléans, Revue environnement, janvier 2006, 9 ; Billet Philippe, Fauchage d'OGM : une relaxe sans nécessité, RD rur. $n^{\circ} 339$, janv. 2006, comm. 36.

${ }^{45}$ A propos de l'arrêt de la Cour de cassation du 7 février 2007 : Darsonville Autrey, La destruction d'OGM ne relève pas de l'état de nécessité, Recueil dalloz 2007 p. 573. 
que sur une éventualité appelée à se développer dans le futur, ne peut en aucun cas être analysée comme un danger actuel et imminent $\gg .{ }^{46}$

Comme le danger n'est pas reconnu, il n'y a pas à en traiter les conséquences. Il est donc logique que la Cour d'Orléans balaie les arguments tirés de l'impossibilité ou de l'ineffectivité des actions en réparation et du recours aux assurances, propres, selon les faucheurs, à conforter l'idée d'une nécessité à agir. Pourtant, l'ineffectivité des actions juridictionnelles préventives ${ }^{47}$ et l'absence d'assurance voulant couvrir le risque OGM $^{48}$ sont réelles et ces carences rendent légitime les interrogations sur la nécessité à agir...

\subsection{L'autorisation préalable vaut preuve scientifique.}

En fondant leur décision sur l'état des connaissances scientifiques, les juges appliquent le même raisonnement que celui qui prévaut en matière de coexistence ${ }^{49}: 1$ 'autorisation permet de réfuter toute action visant à révéler un risque imminent puisque celui-ci a été évalué et jugé inoffensif. En l'absence de démonstration contraire évidente et incontestable lors du procès, il semble donc que la crédibilité scientifique ressorte de l'analyse scientifique réalisée lors de l'autorisation des OGM. Peu importe que des choix politiques contraires aient été faits ultérieurement (application de la clause de sauvegarde ou interdiction administrative de culture OGM); ces choix ne peuvent ni interférer dans la procédure pénale en cours, ni « effacer la responsabilité pénale des prévenus ». Et la raison est simple : les autorisations ne sont données qu'après évaluation des risques; par conséquent le degré de probabilité du risque après autorisation est limité et ne justifie pas d'action collective de destruction. ${ }^{50}$

On comprend alors pourquoi certains considèrent que le processus d'autorisation des OGM proposé par la directive 2001/18 constitue «non plus un rempart contre le danger allégué, mais, au contraire un dangereux outil de propagation des OGM. ${ }^{51}$ On comprend surtout comment les juges ont pu renverser l'un des autres arguments des «faucheurs »- la non-transposition ou la transposition non-conforme de la directive 2001/18 par la France - en considérant que selon la directive, même à considérer qu'il y ait un risque, il ne pourrait fonder une action puisque, suivant la directive 2001/18, le risque « justifie, non la destruction des cultures expérimentales décidée par quelques-uns, mais la précaution, désormais érigée en principe... » et mise en œuvre essentiellement lors de la procédure d'autorisation.

${ }^{46}$ Cour d'appel de Versailles. 9ème chambre n 06/01902. 22 mars 2007 ; Idée développée par Billet Philippe, Fauchage d'OGM : une relaxe sans nécessité, RD rur. n ${ }^{\circ} 339$, janv. 2006, comm. 36.

${ }^{47}$ L Bodiguel, M Cardwell, A Carretero García and D Viti, 'Coexistence of Genetically Modified, Conventional, and Organic Crops in the European Union: National Implementation', L Bodiguel and M Cardwell (eds), The Regulation of Genetically Modified Organisms: Comparative Approaches, op. cit., 163.

${ }^{48}$ L'assurance est pourtant devenu obligatoire avec la loi OGM 2008-595 : Article L663-4 code rural.

${ }^{49}$ L Bodiguel, M Cardwell, A Carretero García and D Viti, 'Coexistence of Genetically Modified, Conventional, and Organic Crops in the European Union: National Implementation', L Bodiguel and M Cardwell (eds), The Regulation of Genetically Modified Organisms: Comparative Approaches, op. cit., 163 (v. plus précisément 168171). V. aussi Bodiguel (Luc), Cardwell (Michael), La coexistence des cultures GM et non GM : approche comparative entre l'Union européenne, le Royaume-Uni et la France, In «Production et consommation durables : de la gouvernance au consommateur citoyen », G. Parent (Dir), Ed. Yvon Blais, 2008, 641 p., 325 366, ISBN : 978-2-89635-193-0.

${ }^{50}$ Solution juges CA Orléans 26 févr. 2008 Sur le délit de destruction du bien d'autrui commis en réunion : «Il est prétendu que les actes ont été symboliques et qu'ils relèvent d'une qualification contraventionnelle seulement. Un tel argument de défense est inopérant dès lors que les prévenus ont agi de manière concertée et préparée. »

${ }^{51}$ Monteillet, op. cit., 64. 
Suivant cette logique, il parait bien difficile pour les faucheurs de trouver une argumentation scientifique. $^{52}$

\subsection{Les principes généraux sont inopérants en matière pénale.}

$\mathrm{Vu}$ la difficulté de faire valoir l'état de nécessité, les faucheurs volontaires, doivent se résoudre à invoquer une série d'arguments qui peuvent donner une impression de grande confusion et qui visent à faire admettre au juge une vision large de l'état de nécessité : un risque de danger hypothétique doit être assimilé à un danger actuel et la protection «d'intérêts généraux » doit fonder la nécessité d'une action collective, organisée, préméditée.

Cette tache - fort ardue puisque l'interprétation jurisprudentielle des motifs d'irresponsabilité pénale est particulièrement stricte - est essentiellement fondée sur l'invocation de principes transcendantaux, qui dépassent largement le droit pénal. Ainsi, l'action des faucheurs est présentée comme un acte nécessaire à la sauvegarde de l'environnement consacré en tant que patrimoine commun des êtres humains dans le préambule de la Charte de l'environnement intégré à la Constitution »; en outre, sont mentionnés deux principes constitutionnels susceptibles d'écarter l'argument d'une atteinte illégitime au droit de propriété : la protection de la santé publique et le principe de précaution sur la base des principes issus de la Convention de sauvegarde des droits de l'homme et des libertés fondamentales (droit à vivre dans un environnement sain), du protocole de Carthagène (précaution), et de la Convention internationale d'Aarhus (droit à l'information du public, à sa participation au processus décisionnel et à l'accès à la justice en matière d'environnement).

Là aussi, la cour refuse de suivre les motifs des faucheurs. Selon elle, ces «principes » ont seulement vocation à « organiser les droits et devoirs de chacun dans le cadre d'un État de droit, » mais ils «ne peuvent être invoqués comme cause d'irresponsabilité pénale par les prévenus qui ont détruit le bien d'autrui. $»{ }^{53}$ Elle montre ainsi son refus de reconnaitre l'irresponsabilité pénale pour la défense d'un patrimoine collectif que serait «l'environnement» ou d'un droit subjectif difficile à identifier et à individualiser dont «l'environnement» serait bénéficiaire ou qui fonderait des actions afin de le protéger ou de protéger la santé ou le droit des personnes à un environnement sain.

Sur le fondement de l'indépendance de la législation pénale, les juges français ont donc confirmé l'indifférence des principes constitutionnels ou internationaux en matière d'état de nécessité. Ils évitent ainsi toute interrogation qui pourraient mettre en cause une lecture strictement pénaliste de l'affaire et qui pourraient être ressentie comme une prise de position politique en matière d'appréciation de la hiérarchie des normes. C'est d'ailleurs ce que certains pensent : si la transcendance des principes était acceptée, le «risque serait de confondre la justification d'une permission juridique - l'état de nécessité - et une opinion exclusivement politique sur le contenu souhaité des règles. ${ }^{54}$

Sur ces deux points, la décision de la Cour d'appel d'Orléans vient d'être « légitimée » par un récent arrêt de la Cour européenne des droits de l'homme (CEDH) ${ }^{55}$ D'une part, les

\footnotetext{
${ }^{52}$ Il reste cependant toujours un doute : et si certains «biogénéticiens » avaient raison...V. Vélot Christian, OGM tout s'explique, Edition Goutte de sable, 2009, 89-191.

${ }^{53}$ CA Orléans 26 févr. 2008. V. Monteillet Sylvain, De la responsabilité pénale des faucheurs jugés à Orléans. Un Etat des lieux du cadre juridique des OGM, Revue juridique de l'environnement (RJE), 2007, p. 56-70.

${ }^{54}$ Monteillet Sylvain, De la responsabilité pénale des faucheurs jugés à Orléans. Un Etat des lieux du cadre juridique des OGM, revue juridique de l'environnement (RJ.E), \&, 2007, p. 56-70

55 v. CEDH, Dec. $5^{\text {e }}$ Sect. 29 juin 2010, Req. $n^{\circ}$ 48629/08, Hubert Caron et autres c. France. Action conduite suite à la condamnation de faucheurs OGM par la Cour d'appel de Versailles. 9ème chambre n 06/01902. 22 mars 2007.
} 
juges de Strasbourg réfutent le bénéfice des articles 2 (droit à la vie ) et 8 (droit au respect de la vie privée et familiale) de la Convention de sauvegarde des droits de l'homme et des libertés fondamentales au motif que les faucheurs n'ont pas démontré « avoir subi directement les effets de la mesure litigieuse » ou être «personnellement affectés, dans leur santé et leur vie privée et familiale, par les OGM cultivés dans les parcelles neutralisées ». D'autre part, ils confirment le caractère inopérant des principes en matière de fait justificatif : « en l'espèce ni l'article 2, ni l'article 8 ne peuvent avoir pour effet d'affranchir les requérants de leur responsabilité pénale pour des actes délictueux ». Toutefois, la Cour pourrait avoir ouvert une brèche en considérant qu'il n'existait pas d'" atteinte au droit de propriété des agriculteurs traditionnels et biologiques résultant de la contamination par les OGM des autres cultures » puisque les faucheurs n'avaient pas fait « valoir que leurs propres cultures ou vignes seraient directement affectées. » Pour certains, cette phrase laisserait penser « que les agriculteurs dont les terres sont voisines de cultures OGM ont un intérêt pour agir en qualité de "victime » d'une potentielle violation de l'article $1^{\mathrm{er}}$ du Protocole $\mathrm{n}^{\circ} 1 .{ }^{56}$

\section{Conclusion}

Au regard des différentes décisions de justice exposées à l'occasion de cet article, il parait possible de dire que, à l'exception des décisions prises par les jurys anglais de la Crown Cour et d'une décision du District Judge, les juges français et britanniques s'inscrivent dans une même vision étroite et légaliste de «ce qui fait droit » dans le domaine des OGM. Loin d'adhérer à la conception défendue par les anti-OGM, ils s'appuient généralement sur une interprétation restrictive des faits justificatifs, sur l'inadéquation des motifs invoqués dans le champ pénal, sur la possibilité de recours préalables aux infractions ou font prévaloir la propriété violée par les faucheurs contre l'éventuel risque de danger pour l'environnement. A ce titre, on peut parler d'une communauté d'esprit. ${ }^{57}$

Certains pourraient penser que cette position cache une désapprobation des actes de désobéissance civile. ${ }^{58}$ On peut se demander en effet si les juges ne simplifient pas trop le discours juridique - ce n'est qu'une question pénale liée à l'état de nécessité - en ne tenant pas compte de la complexité des sources de droit et à leur complexes articulations. ${ }^{59}$ On peut même se s'interroger sur la conception idéologique qu'ils soutiennent consciemment ou non : la réduction volontaire de la portée de principes généraux d'essence internationale qui visent à compenser les risques liés à un marché total. ${ }^{60}$ Pourtant, les théoriciens du droit ont déjà expliqué que si l'obéissance à la loi a une valeur structurelle fondamentale pour les régimes démocratiques, cela n'interdit pas l'existence de contestation active dans certaines situations: «Un citoyen doit obéissance au droit, non à l'opinion d'une personne particulière sur ce qu'est le droit, et il n'agit pas injustement, s'il fait application de sa propre interprétation, réfléchie et raisonnable, de ce qu'exige le droit. (...) cela ne revient pas à dire qu'un individu peut ne tenir aucun compte des jugements des tribunaux. (...) Mais si la question est de celles

\footnotetext{
56 V. Nicolas Hervieu Condamnations pénales à l'encontre des «faucheurs volontaires » d'OGM, http://combatsdroitshomme.blog.lemonde.fr/category/cedh/article-35/ (consulté le 2 sept. 2010).

${ }^{57}$ Nous avons déjà pu observer une situation similaire dans un autre contexte : v. Bodiguel (Luc), Cardwell (Michael), Evolving definitions of agriculture for an evolving agriculture, The Conveyancer and Property Lawyer, sept.-oct. 2005, 419-446 (v ; aussi Revue du marché commun et de l'Union européenne, $\mathrm{n}^{\circ} 490$, juilletaoût 2005, 456-466).

${ }^{58}$ L Bodiguel, 'Conclusion', L Bodiguel and M Cardwell (eds), The Regulation of Genetically Modified Organisms: Comparative Approaches, op. cit., 375.

${ }^{59}$ Morin Edgar, Introduction à la pensée complexe, Seil , 2005, avant propos p. 11.

${ }^{60}$ Supiot Alain, L'esprit de Philadelphie. La justice sociale face au marché total, Seuil, 2010, p. 59-70.
} 
concernant les droits personnels ou politiques fondamentaux (...), un homme ne sort pas de ses droits sociaux, s'il refuse de reconnaitre la décision de la Cour comme un absolu. ${ }^{61}$

Cette conception est largement exprimée par les militants anti-OGM qui arguent de la nécessité d'action - en raison du changement climatique ou des risques de contamination croisée du fait des OGM -, portent le débat sur le terrain politique et social en considérant que le droit en est pour partie l'expression, et utilisent les conflits internes du droit en mettant face à face des législations techniques - en l'espèce, les OGM- ou disciplinaires - le droit pénal en l'espèce - avec des principes qui font de l'environnement une «quasi-personne juridique » dotée de droits subjectifs. Suivant cette approche ouverte du droit sur le monde et les sociétés qui le composent, les désobéissants révèlent « une autre vision du droit (...), faisant apparaitre au grand jour tout ce que procéduralisation et processus d'intériorisation ont alors pour ambition de cadrer : le droit est le produit d'un rapport de force. ${ }^{62}$

\footnotetext{
${ }^{61}$ Dworkin Ronald Prendre les droits au sérieux (chap8) 1968, PUF, 1995 ; p. 316.

${ }^{62}$ David HIEZ, in La désobéissance civile. Approches politiques et juridique, Septentrion, 2008, David Hiez et Bruno Villaba (eds), 70.
} 